\title{
LXVIII. On a new method of treating factorials and figurate numbers
}

\author{
Mr. Peter Nicholson
}

To cite this article: Mr. Peter Nicholson (1819) LXVIII. On a new method of treating factorials and figurate numbers, Philosophical Magazine Series 1, 53:254, 412-418, DOI: $10.1080 / 14786441908652164$

To link to this article: http://dx.doi.org/10.1080/14786441908652164

里 Published online: 23 Jul 2009.

Submit your article to this journal $₫$

Џ Article views: 3

Q View related articles $\square$ 
mated, leaving a very small place for the incisores, which are very small, very short and flat, the two lateral ones on each side are situated diagonally; the second behind, and the two middle ones are only half the size of the others. The tail is bushy, particularly at the top, where there is a white pencil of long hairs; the brown of the remainder is darker than on the body.

From the above accurate description it will appear evident that this animal is very different from the common marten of North America. It must be a very feroeious little animal, which is indicated by the strength of the teeth.

LXVIII. On a new Method of treating Factorials and Figurate Nimbers. By Mr. Peter Nicholson.

To Mr. Tilloch.

Sir, - As the following method of treating factorials and figurate numbers is new, I hope you will have the goodness to insert it in your valuable publication The Philosophical Magazine, as it will be found to apply to many of the most useful parts of algebra: as in the binomial theorem, in equations of all dimensions, in combinations, \&c.

London-street, May 17, 1819.

Peter Nicholson.

\section{FACTORIALs.}

Definition. - An algebraic product of which the difference between every two adjacent factors is equal to the same given number, is called a factorial.

Notation.- In a factorial are to be considered the number of factors, otherwise called the exponent, the first factor, and the common difference, whether + or - .

Let $m$ be the first factor, $n$ the number of factors, and $c$ the common difference; then every factorial may be thus indicated $m^{n \mid c}$ : let $n=4$ and $c=1$, then will $m^{n \mid c}=m^{4 \mid 1}=m(m+1)$ $(m+2)(m+3)$. Again, if $n=5$ and $c=-1$, then will $m^{n \mid c}=$ $\left.m^{5}\right|^{\overline{1}}=m(m-1)(m-2)(m-3)(m-4)$. Again, let $m=-F$, and $c=-e$; then will $m^{n \mid c}=(-p)^{n \mid \tilde{e}}$, which will be affirmative or negative, according as $n$ is even or odd. Thus let $p=3, n=4$, and $e=2$; then $(-3)^{4 / \overline{2}}=(-3)(-5)(-7)(-9)=945$. Again let $n=5$; then $(-3)^{5 / \overline{2}}=(-3)(-5)(-7)(-9)(-11)=-$ 10395.

Proposition.-Any two factorials in which the base of the one is equal to the sum formed by adding the product of the expo- 
nent and common difference of the other to its exponent, may be reduced to one.

For let $m^{n i c}$ and $[m+n c]^{h / c}$ be the two factorials, the base of the latter being formed as announced in the proposition : then because $c$ is the common difference, and $m$ is the first factor of the factorial $m^{n \mid c}$, the second factor will be $m+c$, the third $m+2 c$, the fourth $m+3 c$, and so on. Therefore in $n+1$ factors, the $(n+1)$ th factor from the first will be the first factor, together with $n$ times the common difference $c$; therefore if the factorial $(m+n c)^{h \mid c}$ be annexed to the factorial $m^{n \mid c}$ as two factors, the product will be the factorial $m^{n+h \mid c}$.

PROBLEM.

To resolve a given factorial into two factorial factors, in which the factors of each shall have the same common difference as the factors of the given factorial, and the one a given exponent less than that of the given factorial.

Rule. -1 . Take the less from the greater of the two given exponents, and the remainder will be the exponent of the factorial factor which is not given.

2. To the base of the given factorial apply either of the exponents of the two factorial factors, and the common difference, and the quantity thus formed will be one of the factorial factors.

3 . To the same base add the product of the exponent and common difference of the factorial factor thus completed, and to the sum as a base apply the remaining exponent of the two factorial factors, and the common difference, then the quantity thus formed will be the other factorial factor.

Examples.-1. Resolve $m^{n \mid c}$ into two factorial factors, so that one of them may have the given exponent $r$.

By rule, $n-r$ will be the exponent of the other. Now if $m^{r \mid c}$ be the one factorial, $(m+r c)^{n-r \mid c}$ will be the other.

$\mathrm{Or}$, if $m^{n-r^{\prime} c}$ be the one factorial $[m+(n-r) c]^{r / c}$ will be the other.

2. Resolve $m^{n \mid c}$ into two factorial factors, so that one of them may have the given exponent 1 .

By rule, $n-1$ will be the exponent of the other. Now therefore, if $\left.m^{1}\right]^{c}=m$ be the one factorial factor, then $(m+c)^{n-1 \mid 1}$ will be the other: or if $m^{n-1 / c}$ be the one factorial, then will $[m+(n-1) c]^{1 / c}=m+(n-1) c$ be the other.

3. Resolve $m^{n \mid l}$ into two factorial factors, so that one of them may have the given exponent 1 .

By rule, $n-1$ will be the exponent of the other. If therefore 
$m^{1 / 1}=m$ be the one factorial factor, then will $(m+1)^{n-1 \mid 1}$ be the other. Or if the one factorial factor be $m^{n-1 \mid 1}$, then will the other factorial factor be $(m+n-1)^{1 / 1}=m+n-1$.

4. Resolve $m^{n j c}$ into two factorial factors, so that one of them may have the given exponent $r$.

By rule, $n-r$ will be the exponent of the other. Therefore if $m^{r \mid \bar{c}}$ be the one factorial factor, then will $(n-r c)^{n-r \mid \bar{c}}$ be the other.

Or if $m^{n-r \bar{c}}$ be the one factorial factor, then will [m$(n-r) c]^{r \mid \vec{c}}$ be the other.

5. Resolve $m^{n \mid \overline{1}}$ into two factorial factors, so that one of them may have the given exponent 1 .

By rule, $n-1$ is the exponent of the other. Therefore if $m^{1 \overline{1}}$ $=m$ be the one factorial factor, the other will be $(m-1)^{n-1 \mid \bar{i}}$.

Or, if the one factorial factor be $m^{n-1, \bar{l}}$, the other will be $(m-n+1)^{1 / \overline{1}}=m-n+1$, which is the last term of the factorial $m^{n j \bar{I}}$.

THEORY OF FIGURATE NUMBERS.

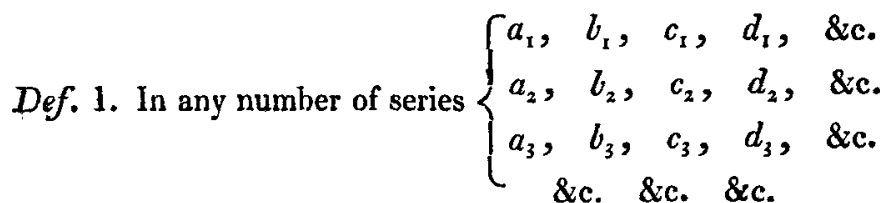

placed in due order, if $n$ be the number of any series beginning with that which is placed first, and $m$ the number of the term in the $n$th series, and if the $m$ th term of the $n$th series be expressed by $\frac{m^{n ! 1}}{l^{n ! 1}}$, each series is called an order of figurate numbers*.

Corollary 1.-Hence by this definition the first order of figurate numbers will be the series of natural numbers $1,2,3,8 c$. : for if in $\frac{m^{n \mid 1}}{1^{n \mid 1}}$ we make $m$ successively equal to $1,2,3, \& c$. and $n$ equal to 1 , we shall have $\frac{1^{1 \mid 1}}{1^{1 \mid l}}, \frac{2^{1 \mid 1}}{1^{1 \mid 1}}, \frac{3^{1 \mid 1}}{1^{111}} \& c$., which are the same as the numbers $1,2,3,8 c$.

* The author has here adopted Legendre's definition of figurate numbers.

Def. 
Def. 2.-The $m$ th term of the first, second, third, \&c. order is called the $m$ th vertical column.

Thus $\frac{m^{1 \mid 1}}{1^{1 \mid 1}}, \frac{m^{2 \mid 1}}{1^{2 \mid 1}}, \frac{m^{3 \mid 1}}{1^{3 / 1}}, \& \mathrm{c}$. is the $m$ th vertical column.

Corollary.-Hence the $n$th term of the $m$ th vertical column is the same as the $m$ th term of the $n$th order of figurate numbers; for by definition $1, \frac{m^{n ! 1}}{1^{n ! 1}}$ is the $m$ th term of the $n$th order, and by the last definition the very same is the $n$th term of the $m$ th vertical column.

Def.3.-The first term of the $n$th order, the second term of the $(n-1)$ th order, the third term of the $(n-2)$ th order, \& $\mathrm{c}$, is called the $n$th diagonal series.

Thus $\frac{1^{n: 1}}{1^{n \mid 1}}, \frac{2^{n-1 \mid 1}}{1^{n-1 \mid 1}}, \frac{3^{n-2 \mid 1}}{1^{n-2 \mid 1}}$, \&c. is the $n$th diagonal series.

Corollary 1.-Hence if $x$ be the number of the term of a diagonal series, then any term will be $\frac{x^{n-x+1} \mid 1}{1^{n-x+1 \mid 1}}$, where $x$ must never exceed $n+1$.

Corollary 2.- Hence if $n$ be made equal to 1 , and $x$ successively equal to 1 and 2 , the first diagonal series will be $\frac{1^{1-1+1: 1}}{1^{1-1+1 \mid 1}}, \frac{2^{1-2+1_{1} 1}}{1^{1-2+11^{1}}}$; that is, $\frac{1^{1 / 1}}{1^{1 ! 1}}, \frac{2^{0 \mid 1}}{1^{0 \mid 1}}$, which in effect is the same as 1,1 .

Corollary 3.-Hence the $m$ th terms of any two consecutive orders of figurate numbers will also be the $m$ th terms of two consecutive diagonal series.

Proposition 1.-The $(m+1)$ th term of the $(n+1)$ th order is equal to the $m$ th term of the $(n+1)$ th order and the $(m+1)$ th term of the $n$th order.

For $\frac{(m+1)^{n+1 \mid 1}}{1^{n+1 \mid 1}}$ is the $(m+1)$ th term of the $(n+1)$ th order.

Now each of the terms of the fraction $\frac{(m+1)^{n+1 / 1}}{1^{n+1 \mid 1}}$ may be resolved into two factorial factors, so that one of them may have the given exponent 1 : therefore the factorial $(m+1)^{n+1 \mid l}$ is equal to $(m+1)^{n \mid 1} \times[m+(n+1)]=m^{n+1 \mid 1}+(n+1)(m+1)^{n \mid 1}$, and the factorial $1^{n+1 / 1}=1^{n \mid 1} \times(n+1)$ : whence 
$\frac{(m+1)^{n \mid 1}}{1^{n \mid l}}$

$\frac{(m+1)^{n+1 \mid l}}{1^{n+1 \mid 1}}=\frac{m^{n+1 \mid l}+(n+1)(m+1)^{n \mid 1}}{1^{n \mid 1} \times(n+1)}=\frac{m^{n+1} \mid 1}{1^{n+1 \mid 1}}+$

Proposition 2.-In any two consecutive orders of figurate numbers, the sum of $m$ terms of the antecedent order is equal to the $m$ th term of the consequent order.

Let $1, b, c, d, \&$. $1, \beta, \gamma, \delta, \& c$.

be any two consecutive orders of figurate numbers; than by the last proposition

$$
\begin{gathered}
1+b=\beta \\
\beta+c=\gamma \\
\gamma+d=\delta \\
\& c . \quad \& \mathrm{c} .
\end{gathered}
$$

The sum of these equations is

$$
1+\beta+\gamma+b+c+d=\beta+\gamma+\delta
$$

take away the common quantities $\beta, \gamma$, and there will remain

$$
\mathbf{1}+b+c+d=\delta \text {; Q. E. D. }
$$

Corollary 1.-Hence the sum of $m$ terms of any order is equal to the $m$ th term of that order, when the exponent of each of its terms is increased by unity.

Corollary 2.-Hence the first order of figurate numbers being given, the consecutive orders may be derived to any order required; thus,

$\begin{array}{lllrrrr}\text { lst order } & 1 & 2 & 3 & 4 & 5 & 6 \\ \text { 2d order } & 1 & 3 & 6 & 10 & 15 & 21 \\ \text { 3d order } & 1 & 4 & 10 & 20 & 35 & 56 \\ \text { 4th order } & 1 & 5 & 15 & 35 & 70 & 126\end{array}$

$\& \mathrm{c}$.

\&c.

Proposition 3.-The $m$ th term of the $n$th order is equal to the $(n+1)$ th term of the $(n-1)$ th order.

For $\frac{m^{n \mid 1}}{1^{n \mid 1}}$ is the $m$ th term of the $n$th order of figurate num-

bers. Now the factorial $m^{n \mid l}$ may be resolved into two factorial factors, so that one of them may have the given exponent $m-1$ (see factorials): therefore $\left.m^{n}\right|^{1}=m^{n-m+\left.1\right|^{1}} \times(n+1)^{m-1 / 1}$; also the factorial $1^{n \mid l}$ may be resolved into two factors, so that one of them may have the given exponent $n-m+1$, therefore

$$
\begin{aligned}
& 1^{n \mid 1}=1^{m-1 \mid 1} \times m^{n-m+1 \mid 1} \\
& \text { whence } \frac{m^{n \mid 1}}{1^{n \mid 1}}=\frac{m^{n-m+1 \mid 1} \times(n+1)^{m-1 \mid 1}}{1^{m-1 \mid 1} \times m^{n-m+1} \mid 1}=\frac{(n+1)^{m-1 \mid 1}}{1^{m-1 \mid 1}} .
\end{aligned}
$$


Corollary 1.-Hence one expression of figurate numbers can easily be converted into another equivalent expression by the following.

Rule.-Add unity to the exponent of the numerator of the given expression, and it will give the first factor of the numerator of the new expression; and take unity from the first factor of the numerator, and the remainder will be the exponent of both the numerator and denominator of the new expression; the first factor of the denominator being the same as that of the given expression.

Proposition 4.-The first, second, third, \&c. terms of the $m$ th vertical column are equivalent to the second, third, fourth, \&c. terms of the $(m-1)$ th order of figurate numbers.

For by definition the first, second, third, fourth, \&c. terms of the $(m-1)$ th order of figurate numbers are respectively $\frac{1^{m-111}}{1^{m-111}}$. $\frac{2^{m-1 \| 1}}{1^{m-1 \mid 1}}, \frac{3^{m-1 \mid 1}}{1^{m-1 \mid 1}}, \frac{4^{m-1 \mid 1}}{1^{m-1 \mid 1}}$, \&c. : then by the rule to corollary 1 , proposition 3 , if the exponents of each factorial in the numerator be increased by 1 , and the first factor of each of the factorials be diminished by 1 for the first factor and exponent of the respective terms of a new series of figurate numbers, we shall have the equivalent series $\frac{m^{0 \mid 1}}{1^{0 \mid 1}}, \frac{m^{1 \mid l}}{1^{1 \mid 1}}, \frac{m^{* 11}}{1^{3 \mid 1}}, \frac{m^{3 \mid 1}}{1^{3 \mid 1}}, \& c . ;$ but by definition 2, the second, third, fourth, \&c. terms $\frac{m^{1 \mid l}}{1^{111}}, \frac{m^{2 \mid l}}{1^{211}}$, $\frac{m^{311}}{1^{311}}, \& c$. are called the first, second, third, \&c. terms of the $m$ th vertical column. Q. E. D.

Corollary.-Hence because by proposition 2 , the sum of $m$ terms of the preceding order of any two consecutive orders of figurate numbers is equal to the $m$ th term of the consequent order; the sum of $m$ terms of the $(m-1)$ th vertical column must be less by unity than the sum of $m$ terms of the $(m-1)$ th order of figurate numbers.

Proposition 5.-The $n$th diagonal series of figurate numbers is $1, n, \frac{n^{211}}{1^{\text {211 }}}, \frac{n^{311}}{1^{\text {211 }}}$, \&c.

For by corollary to definition third, any term of the $n$th diagonal series is $\frac{x^{n-x+111}}{1^{n-x+111}}$.

Vol. 53. No. 254. June 1819.

$\mathrm{Dd}$

But 
But by the principles of factorials $\frac{x^{n-x+111}}{1^{n-x+1 \mid 1}}=\frac{n^{n-x+1 \mid 1}}{1^{n-x+1 ! 1}}$, where the first factor in the numerator of the second side of the equation is the last factor of the numerator in the first side; and because the common. difference of the factors of the first side is +1 , the common difference of the factors in the second side must be -1 ; also because the reverting of the fraction does not change the number of factors, therefore the exponent of the numerator must be the same on both sides, as is exhibited.

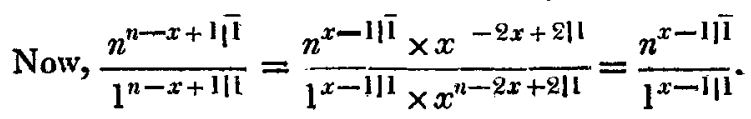

Let $x$ be expounded by $1,2,3,8 x$. in the last side of this equation, and we shall have $1, n, \frac{n^{2 \bar{l}}}{1^{2|1|}}, \frac{n^{3 / \bar{l}}}{1^{3 \mid \bar{l}}}$, \&c. Q. E. D.

Proposition 6.-The sum of any two consecutive terms $x$ and $x+1$ of the $n$th diagonal series of figurate numbers is equal to the $(x+1)$ th term of $(n+1)$ th diagonal series. For, by Proposition 1 , the sum of the $m$ th term of the $(n+1)$ th order, and the $(m+1)$ th term of the $n$th order is equal to the $(m+1)$ th term of the $(n+1)$ th order. Now the $m$ th term of the $(n+1)$ th order and the $(m+1)$ th term of the $n$th order are any two consecutive terms $x$ and $x+1$ of any diagonal series $n$; also the $(m+1)$ th term of the $(n+1)$ th order is the $(x+1)$ th term in the next diagonal series following. Q. E. D.

Corollary 1.-Hence if any diagonal series be given, the next following will be found: Thus, let $1, B, C, D, \& c$. be given, then the next will be $1,(1+B),(B+C),(C+D)$, \&c.

Corollary 2.-Hence if the first diagonal series be given, we may derive as many consecutive diagonal series as we please, as in the following table:

First diagonal series

Second diagonal series.

Third diagonal series

Fourth diagonal series

Fifth diagonal series

\&c.

$\& \mathrm{c}$. 